\title{
Venture Capital im Umbruch
}

\author{
Michael Groß
}

Der Boom, dem der Venture Capital Markt in der Vergangenheit jährliche Zuwachsraten in Höhe von dreistelligen Millionenbeträgen zu verdanken hatte, ist in den Jahren 2001 und 2002 den Erwartungen entsprechend in eine Konsolidierungsphase übergegangen. So lassen sich anhand der vom Bundesverband deutscher Kapitalbeteiligungsgesellschaften (BVK) veröffentlichten Zahlen nicht nur eine Stabilisierung, sondern auch im Jahr 2002 eine Reduzierung der Investvolumina und eine Veränderung des Investitionsverhaltens erkennen. Die Bruttoinvestitionen der BVK-Mitglieder, welche nach eigenen Angaben $90 \%$ des gesamten Marktvolumens ausmachen, betrugen 2001 ca. 4,4 Mio. Euro und lagen damit auf dem Niveau des Vorjahres. Davon wurden $80 \%$ in Form von klassischem Eigenkapital, 19,6 \% als eigenkapitalähnliche Mittel, z. B. in Form von mezzaninen Finanzmitteln vergeben. Bezuiglich der bevorzugten Branchen verlagerte sich der Fokus von den Hightech-Firmen wieder stärker zu den traditionellen Segmenten, so sank der Anteil der Bruttoinvestitionen in den Bereichen Computer und Kommunikationstechnologien gegenüber dem Jahr 2000 um 12,4\% auf 24,6\%. ${ }^{1}$ Dieser Trend wird sich auch in den Jahren 2002 und 2003 fortsetzen, ein Ende der Konsolidierungsphase wird in einem Zeitraum zwischen 12 und 24 Monaten erwarten.

Die Verschiebung innerhalb der Branchen ist auf die Risikoverlagerung von technologieorientierten Frühphasenfinanzierungen hin zu branchenoffenen Wachstumsfinanzierungen und Buy-outs zurückzuführen. So sank im Bereich der Early-stage-Finanzierungen die Investitionssumme gegenuiber dem Jahr 2000 von 1,6 Mrd. Euro auf 1,2 Mrd. Euro. Ebenso verringerte sich der Anteil der Expansions-Finanzierungen an den Bruttoinvestitionen im Vergleich zum Vorjahr. Hingegen konnte sich der Anteil der Buy-out-Finanzierungen mehr als verdoppeln und liegt bei insgesamt 37,3\%. Ursache dafür sind u. a. Strukturveränderungen und Konsolidierungsmaßnahmen innerhalb der Wirtschaftsunternehmen, die dazu passende Finanzierungsformen nach sich ziehen.

Der Traum vom schnellen Geld, der in den letzten Jahren, auch ausgelöst durch die Euphorie am „Neuen Markt“, zahlreiche neue Venture Capital (VC)-Gesellschaften auf den Plan rief, platzte für viele dieser Gesellschaften in Form von erheblichen Wertberichtigungen und Abschreibungen. Dabei wirken sich die zwischen 1998 und $2001 \mathrm{zu}$ verzeichnenden Überbewertungen von Hightech-Unternehmen besonders negativ aus. Die neuen Schlagworte in der VC-Branche heißen Konsolidierung und Schadensbegrenzung. So stieg 2001, laut
BVK, der Anteil der Totalverluste bei den Abgängen auf $36 \%$ und stellte somit den bedeutendsten Exitkanal dar. Der Branchenfokus, der in den letzten Jahren einseitig auf die New Economy gerichtet war, bewirkte, dass das zur Verfuigung stehende Kapital zu schnell in junge, aufstrebende Unternehmen investiert wurde, ohne die VCuiblichen Kriterien der „Due diligence“ im Sinne einer sorgfältigen Prüfung der Entwicklungsmöglichkeiten der einzelnen Investments zu beachten. Einige der neuen „Kapitalzuwanderer“ erlebten und erleben noch ihr Waterloo, die qualifizierten VC-Geber kämpfen um ihre Portfolios und Marktpositionen im Rahmen eines in Deutschland noch nicht erlebten Konsolidierungsprozesses.

So erklärte Raymond Lane, ehemaliger Chief Operating Officer bei Oracle und jetzt General Partner bei der Risikokapitalfirma Kleiner Perkins Caulfield \& Byers, in einem Interview der Wirtschaftswoche ${ }^{2}$, dass die gesamte Informationstechnologie (IT)-Branche in Folge der überhöhten Bewertungen an Glaubwürdigkeit verloren habe, ihr aber der momentane Druck, unter dem sie steht, helfen wird diese wiederherzustellen. Denn Firmen müssen nun innerhalb von wenigen Monaten mit knappen Budgets beweisen, dass sie echte Wettbewerbsvorteile bringen können. Diese Aussage gilt aus Sicht des Autors auch für den Biotech-Sektor.

Die damit in engem Zusammenhang stehende schlechte Stimmung an den Börsen sollte man dabei auch auf die Verweigerung der breiten Kapitalanlegerschaft gegenüber gescheiterten Managementstrategien zurüickführen, was zur Sperrung des attraktiven Exitkanals für die VC-Geber fuihrt. Es fehlen zum Teil Bar-Reserven, die die VC-Geber dringend für portfolioerhaltende Folgeinvestitionen benötigen. War es in den vergangenen Jahren möglich enorme finanzielle Mittel einzuwerben, so ist auch diese Möglichkeit zur Einwerbung von Geldmitteln gegenwärtig durch die allgemeine Zurüickhaltung und schlechte Konjunkturlage eingeschränkt. Alle haben begriffen, dass die Risiken für Investments in Technologieunternehmen real höher sind als die Chancen, trotzdem braucht der Standort Deutschland genau diese Art von neuen Unternehmen.

Der Umbruch, in der sich der Venture Capital-Markt zur Zeit befindet, ist somit nicht als Ende, sondern vielmehr als der Anfang einer neuen Qualität und Chance anzusehen. Dabei ist der Fruihphasenfinanzierung eine besondere Bedeutung beizumessen um einen Ansatzpunkt fuir neue wettbewerbsfähige Jobs in Technologieunternehmen und für deren Expansion zu schaffen. In dieser 
Phase ist es generell wichtig den jungen Unternehmen nicht nur die finanziellen Mittel zu Verfügung zu stellen, sondern sie zusätzlich durch qualifizierte Beratung in allen Unternehmensbereichen zu unterstuitzen. Die Entwicklung eines neuen, innovativen Produktes ist nur der erste Schritt, denn nur eine sich daran anschließende erfolgreiche Markteinfuihrung ist der Garant zur Erwirtschaftung dauerhafter Erträge. Ziel sollte die Sichtung und Vorbereitung der neuen Unternehmer und die Streuung des Risikos sein, um die Möglichkeit für Beteiligungen an neuen, vielversprechenden Investments mit einer hohen volkswirtschaftlichen Bedeutung zu schaffen.

Die Hightech-Unternehmen, die unter der Krise leiden, müssen sich den neuen Anforderungen und gegenwärtigen Erwartungen stellen. Das gilt auch und insbesondere fuir die Biotechnologie, die in Zukunft auf Grund der Verzahnung von Grundlagenforschung an wissenschaftlichen Einrichtungen und Technologieentwicklung in den Unternehmen erfolgversprechende Investitionsmöglichkeiten bietet. Aber auch hier dürfen die Risiken, die mit der Umsetzung einer durchaus hervorragenden Geschäftsidee verbunden sind, nicht unterschätzt werden. Hat das Platzen der „Seifenblase“ Neuer Markt doch eindrucksvoll gezeigt, dass eine Bewertung auf Grund überzogener Erwartungen zwar kurzfristig einen enormen Hype auslösen kann, mittel- und langfristig gesehen jedoch eine verlustreiche Neubewertung unumgänglich ist. Dabei ist zu beachten, dass der erforderliche Kapitalbedarf fuir Biotech-Unternehmen höher anzusetzen ist, als z. B. bei IT-Unternehmen. Kostet der Aufbau eines ITUnternehmens zwischen 10 bis 20 Mio. Euro, so steigt dieser Betrag bei Biotech-Unternehmen je nach Produktzielstellung auf das 2- bis 10-fache. Trotz der Risiken bleibt, nach Befragung von ca. 400 europäischen VCManagern anlässlich der evca-Technologiekonferenz in Barcelona im Oktober 2002, der Biotech-Sektor einer der wesentlichen VC-Investitionsbereiche neben ICT und Nanotech.

Auch die Mittelstandsfinanzierung, so zeigt eine neue Studie des Sparkassen- und Giroverbandes ${ }^{3}$, ist von der momentan schwachen Konjunkturlage betroffen. Die Eigenkapitalausstattung des Mittelstandes ist über alle Wirtschaftsbereiche hinweg mangelhaft. Dies fuihrt zwangsläufig zu einem Rückgang sowohl der Kapitalzufuhr als auch der Investitionen. Neben der Eigenkapitalquote geben weitere Kennzahlen wie die Umsatzrentabilität, Gesamtkapitalverzinsungsquote sowie Personalaufwandsquote Grund zur Sorge. Dabei wird die Lage der kleinen und mittleren Unternehmen (KMU) in den neuen Bundesländern noch pessimistischer eingeschätzt. Das heißt, auch der Mittelstand bedarf einer gezielten Eigenkapital-Unterstuitzung und Förderung von Seiten des Bundes und der Länder, die ihren Anschubcharakter gerade hier verstärken müssen.

Insofern sollten Garantie- und Bürgschaftsprogramme des Bundes und der Länder nicht als Rettungsanker für Einzelne, sondern als Motor für die dringend benötigten innovativen Kleinst-, Klein- und Mittelstandsunternehmen eingesetzt werden. Der in der Praxis zu ver- zeichnende Abbau der Risikoreduzierung für qualifizierte VC-Geber und Privatinvestoren durch Bund und Länder sowie ein zu starkes Festhalten an der Zuschussmentalität werden eine Kapitalabwanderung der VCGeber aus dem real hochrisikoreichen und gleichzeitig mit enormen Chancen ausgestatteten Frühphasenfinanzierungsgeschäft bewirken. Das nachfolgende Expansionsgeschäft wird abnehmen, da dort immer weniger neue innovative Unternehmen ankommen.

\section{Anmerkungen}

1 BVK-Statistik 2001

2 „Der Druck hilft der Branche“, in: Wirtschaftswoche, $\mathrm{Nr}$. 15, 2002, S. $80 \mathrm{f}$.

3 Martin Erkel (InnovationsErfolg KG): ,Diagnose Mittelstand“, 2002

\author{
Autor \\ Prof. Dr. Michael Groß \\ Geschäftsfuihrer \\ BC Brandenburg Capital GmbH \\ Im Technologiepark 1, 15236 Frankfurt (Oder) \\ Internet: www.bc-capital.de \\ Tel. (03 35) 557-1690 \\ Fax (03 35) 557-1699 \\ E-Mail: gross@bc-capital.de
}

